\title{
To the mean-field theory of a two-sublattice antiferromagnet
}

\author{
A.A. Zvyagin ${ }^{1,2}$ and G.A. Skorobagat'ko ${ }^{2}$ \\ ${ }^{1}$ Max-Planck-Institut für Physik komplexer Systeme, Dresden, D-01187, Germany \\ ${ }^{2}$ B. Verkin Institute for Low Temperature Physics and Engineering of the National Academe \\ of Sciences of Ukraine, 47 Lenin Ave., Kharkov, 61103, Ukraine \\ E-mail: zvygin@ilt.kharkov.ua
}

Received March 1, 2005, revised January 10, 2006

\begin{abstract}
Numerical solutions of the mean-field theory of a two-sublattice antiferromagnet with a strong crystalline electric field are studied. The validity of the solutions was checked with the help of known analytical results for the special case of the lowest doublet $(S=1 / 2)$. For larger values of the lowest multiplet $(S>1 / 2)$ the temperature and magnetic field dependencies of the antiferromagnetic order parameter and the total magnetization are calculated and their features are analyzed. We predict new re-entrant phase transitions between Néel and paramagnetic phases for $S \geq 1$.
\end{abstract}

PACS: 75.10.-b, 75.30.Kz, 75.50.Ee

Keywords: magnetic sublattice, mean-field approximation, re-entrant phase transitions.

Antiferromagnetism was discovered theoretically more than 80 years ago by Néel and Landau [1,2]. Later their theoretical prediction was checked experimentally, see, e.g., [3]. Since that time many antiferromagnets were discovered experimentally. According to the theories of Néel and Landau, antiferromagnetism is characterized by at least two magnetic sublattices (compared to only one sublattice in the case of the ferromagnetism). Each of those sublattices has a spontaneous magnetic moment in ordered phase(s). However, the total magnetization of a system is zero in the antiferromagnetic phase. The number and the symmetry of the magnetic sublattices is determined by the symmetry of the crystal and by the chemical nature of the compound. For the simplest example of a two-sublattice antiferromagnet with equivalent sublattices the antiferromagnetic order parameter is the vector of antiferromagnetism, $\mathbf{l}=(1 / 2)\left(\mathbf{m}_{1}-\mathbf{m}_{2}\right)$, where $\mathbf{m}_{1,2}$ are the magnetizations of each of the sublattices. In these notations the sum of the magnetizations of the sublattices $\mathbf{m}=(1 / 2)\left(\mathbf{m}_{1}+\mathbf{m}_{2}\right)$ characterizes possible phases of the two-sublattice antiferromagnet which have nonzero total magnetization.

Probably the most useful and known method for describing theoretically the magnetic phase transitions of a multi-sublattice antiferromagnet is the mean-field approximation [4]. It must be emphasized that the mean-field approximation does not work well for low temperatures in the ordered phase(s) for standard Heisenberg magnets with (almost) magnetically isotropic interactions, where one has to use the spin-wave description [5]. Let us consider the Hamiltonian of a bi-partite magnetic structure

$$
\begin{gathered}
\mathcal{H}=-H\left(J_{1}^{z}+J_{2}^{z}\right)+ \\
+A_{1} \sum_{n, m}\left(\mathbf{j}_{1, n} \mathbf{j}_{1, m}+\mathbf{j}_{2, n} \mathbf{j}_{2, m}\right)-A_{2} \sum_{n, m} \mathbf{j}_{1, n} \mathbf{j}_{2, m},
\end{gathered}
$$

where $H \equiv g_{J} \mu_{B} h, h$ is an external magnetic field, here supposed to be directed along the $z$ axis (with $g_{J}$ being the $g$-factor of a magnetic ion, and $\mu_{B}$ being the Bohr magneton), $J_{1,2}^{z}=\sum_{m} j_{1,2, m}^{z}$ are operators of $z$-projections of the total moments of sublattices, enumerated by indices 1 and 2, respectively. Here we consider the simple case of equivalent magnetic sublattices, i.e., the magnetic moments have similar values $\left\langle\mathbf{j}_{1,2}^{2}\right\rangle=j(j+1)$ in each sublattice. $A_{1}$ denotes the interaction between magnetic moments belonging to the same magnetic sublattice, $A_{2}$ is the interaction between magnetic moments belonging to different sublattices, and the summations are over the co-ordination spheres of the interaction of moments belonging to each sublattice and between sublattices, respectively. In this study we consider the case $A_{1,2} \geq 0$. The 
case with positive values of $A_{1}$ and negative values of $A_{2}$ corresponds to a ferromagnet. The situation with positive $A_{2}$ and negative $A_{1}$ is known to produce more than two magnetic sublattices. Finally, the case with $A_{1,2} \leq 0$ can be considered analogously to that of $A_{1,2} \geq 0$ with the re-definition of magnetic sublattices.

In the mean-field approximation one replaces the operator $\sum n, m \mathbf{j}_{1,2, n} \mathbf{j}_{1,2, m}$ by $(z / 2) \mathbf{m}_{1,2} \sum \mathbf{j}_{1,2, n}+N m_{1,2}^{2} / 2$, where $N$ is the number of sites, $z$ is the co-ordination number, $\mathbf{m}_{1,2} \equiv(2 / N)\left\langle\sum \mathbf{j}_{1,2, n}\right\rangle$ are the average values of magnetizations per site of each sublattice. Obviously, in this approximation the Hamiltonian Eq. (1) gets the form

$$
\begin{gathered}
\mathcal{H} \approx-\left(H+H_{1}\right) J_{1}^{z}-\left(H+H_{2}\right) J_{2}^{z}- \\
-\sum_{x, y}\left(H_{1}^{x, y} J_{1}^{x, y}+H_{2}^{x, y} J_{2}^{x, y}\right)+\text { Const }
\end{gathered}
$$

where $H_{1,2}^{x, y, z}=J_{1} m_{1,2}^{x, y, z}-J_{2} m_{2,1}^{x, y, z}$ are the effective fields, which act on moment of the system from other moments. Notice that $J_{1,2}$ are parameters of the mean-field interaction between magnetic moments of each sublattice and between sublattices, respectively (they are obviously related to the constants $A_{1,2}$ ). Generally speaking, the consideration of the Hamiltonian Eq. (2) instead of Eq. (1) requires the fulfilment of six self-consistency conditions (which are the equations for the determination of $m_{1,2}^{x, y, z}$ in the mean-field approximation). In the absence of the external magnetic field, $h=0$, the solution of these mean-field equations yields the Néel ground state with antiparallel to each other magnetizations of two magnetic sublattices. The degeneracy of the direction of the vectors of magnetization (i.e., of the vector of antiferromagnetism) is broken, if one first switches on the external field, and, then, considers the limit $h \rightarrow 0$, as usually. For nonzero temperatures, especially for temperatures of the order of $J_{1,2}$ (we use temperature units for values of any energies for simplicity), these mean-field equations are nonlinear ones, and it is very difficult, and often even impossible, to solve them explicitly. However, for some real systems one can take into account special conditions, e.g., the symmetry of a crystal and the nature of magnetic moments. Using those conditions one can a priori find some of values of $m_{1,2}^{x, y, z}$. For example, for many rare-earth compounds the crystalline electric field of nonmagnetic ligands splits the ground-state multiplet of electrons of the $4 f$ shell with the degeneracy $2 j+1$ into several multiplets of lower degeneracies. One can take into account the large value of the energy splitting $E_{\text {spl }}$ between the lowest crystalline electric field multiplet, in comparison with the characteristic values of coupling constants $J_{1,2}$ and the applied external magnetic field $h$. Then, for temperatures $T \leq E_{\mathrm{spl}}$ instead of the initial system, consisting of moments of values $j$, we can approximately consider a simpler, from the theoretical viewpoint, system of effective $(2 S+1)$-level systems (where $2 S+1$ is the degeneracy of the lowest crystalline electric field multiplet). Due to such an approximation, one has to consider anisotropic effective $g$-factors of the system of effective moments, i.e., the situation of effective «easy-axis» magnets (with the projections of effective $g$-factors on one direction, the easy axis, being much larger than other projections). It is not difficult to show that the contribution of the components perpendicular to the easy axis is small. If we apply, for simplicity, the external magnetic field along the distinguished axis (say, $z$ ), then the contribution of $H_{1,2}^{x, y}$ to the energy in the main order in $1 / E_{\mathrm{spl}}$ is $\left(H_{1,2}^{x, y}\right)^{2} / E_{\mathrm{spl}}$, see, e.g., [7], and can be neglected in the first order in $E_{\mathrm{spl}}^{-1}$. In fact, it corresponds to the Van Vleck contribution (which does not depend on the temperature) $[9,10]$. Hence, in this situation, in the main order in $E_{\mathrm{spl}}^{-1}$ we can neglect those contributions, using for temperatures $T \ll E_{\mathrm{spl}}$ the solution of mean-field equations $H_{1,2}^{x, y}=0$, which implies the «easy-axis» behavior of magnetic moments in such rare-earth compounds. Similar Ising-like approach was used to the theoretical description of transition metal antiferromagnets, e.g., of the ferrous carbonate $\left(\mathrm{FeCO}_{3}\right)$; a recent review can be found in [11]. Hence, in this case the mean-field equations have the form

$$
\begin{aligned}
& m_{1}=S B_{S}\left[S\left(H+J_{1} m_{1}-J_{2} m_{2}\right) / T\right], \\
& m_{2}=S B_{S}\left[S\left(H+J_{1} m_{2}-J_{2} m_{1}\right) / T\right],
\end{aligned}
$$

where

$$
B_{S}(x)=\frac{2 S+1}{2 S} \operatorname{coth}\left(\frac{2 S+1}{2 S} x\right)-\frac{1}{2 S} \operatorname{coth}\left(\frac{y}{2 S}\right)
$$

is the Brillouin function. Notice, that in our simplified approach of effective moments we have only one projection of $\mathbf{m}_{1,2}$, namely $m_{1,2}^{z} \equiv m_{1,2}$ (as well as we shall use the notations $m$ and $l$ instead of $\mathbf{m}$ and $\mathbf{l}$ in what follows). Observe that Eqs. (3) in the ground state for $H=0$ yield the Néel antiferomagnetic ordering with the (nonzero) spontaneous magnetizations of sublattices being antiparallel to each other, while the total magnetization is zero.

Unfortunately, the set of equations (3) cannot be solved explicitly for any values of $H, J_{1,2}$, and $T$ for any value of $S$. Some simple answers can be obtained in the limiting cases: in the ground state, at high enough temperatures, $T \gg H, J_{1,2}$ and for $S=1 / 2$ [6-8]. In the general case, unfortunately, there are no results for the 
behavior of the antiferromagnetic order parameters and magnetizations as functions of the temperature and external magnetic field. In our work we solved numerically Eqs. (3) for the definite in each case set of values of the external magnetic field $h$ and temperature $T$. Namely, for fixed values of the coupling constants $J_{1}$ and $J_{2}$ each time the procedure of such the calculations started from one of the two points: $T_{1,2}=T_{1 \min , 2 \mathrm{~min}}$ (with the fixed value of $H$ ), or $H_{1,2}=0$ (with the fixed value of $T$ ). For these points one expects to have the following reasonable conditions: $\left|l_{1 \min , 2 \min }\right|=|S|$, and $m_{1 \min , 2 \min }=0$ (we chose those values positive), which does not violate the generality of our approach (see below), i.e., we start from the Néel antiferromagnetically ordered low-temperature phase. Indeed, for all considered magnetic field dependencies of $l$ and $m$, at some fixed $T \leq T_{N}$, where $T_{N}$ is the Néel temperature (the temperature of transition to paramagnetic phase with $l=0)$, the starting point $(0 ; T)$ of calculations in the «plane» $H-T$ corresponds to the Néel antiferromagnetic low-temperature phase, with $m \approx 0$, and it was confirmed by both analytical and our numerical results for the case $H=0$. On the other hand, for the temperature dependencies of $l$ and $m$ with definite fixed values of the external magnetic field $h$, if $T$ is small enough (more exactly: $T \approx 0$ ), the «starting points» of our numerical calculations (points, like $\left.\left(H ; T_{\min }\right)\right)$ are situated in the region of instability, where the first order phase transitions take place (in accordance with analytical [7], and our numerical results mentioned above). It is evident also from more obvious reasons, since the main-field approach doesn't work well at $T \approx 0$ for Heisenberg magnets. So, the initial conditions for our numerical calculations of $l, m$, taken in this region $\left(T \approx T_{\min }\right)$, are inessential for other regions, where $T \gg T_{\min }$, because of the uncertainty of such temperature dependencies at $T$ close to 0 . Indeed, this uncertainty is reduced to the «independence» of considered temperature dependencies of the «initial conditions», required in our calculation procedure. It was realized at quite total interval of possible values of $T$, except the small region near the point $T=0$. We also have checked and confirmed this fact in our present calculations. That is why, one can speak about the «stability» of the obtained results with respect to variations of the «initial conditions», necessary for our numerical processing. Also, hence, it means that the Néel antiferromagnetic low-temperature phase with $\left|l_{1 \min , 2 \min }\right|=|S|$ and $m_{1 \min , 2 \min }=0$ is the state, realistic enough for our purposes, and we have chosen reasonable initial conditions for our numerical processing. Starting from those «initial» data (which, though, basically did not affect the final result) the calculation program step by step (in two taken up cycles) passed the total needed region of parameters $H$ or $T$ and $J_{1}$ or $J_{2}$ (whereas $T$ or, accordingly, $H$ were fixed in «plane» $H-T)$, calculating for those points the values $m$ and $l$. Naturally, such calculations are approximate, and the accuracy of them has to be dependent of the smallness of the steps of changes of $T$ and $H$ and of the validity of the «initial» conditions for $m$ and $l$ (see above). However, the performed calculations have shown the stability of the obtained results with respect to the changes of the values of steps and «initial» conditions. This fact also tells us about the acceptable accuracy of the calculation scheme, used in our problem.

To check the validity of our numerical calculations we first compare our numerical results with the analytical ones, which, unfortunately, are known only for the case $S=1 / 2$. Previous studies of the mean-field solutions for the considered model were limited either to low-temperature or to high-temperature behavior only, or they used the smallness of the antiferromagnetic order parameter $|l| \ll<1 / 2$ [6-8]. In fact, despite the long history of the problem, very little was known about the (probably most interesting) situation, for values of the temperature and the external magnetic field which are comparable to or smaller than the values of the intra- and inter-sublattice coupling constants. From Refs. 6, 7 it is known that the behavior of the system depends on the values of the coupling constants $J_{1}$ and $J_{2}$. Namely, for the fixed value of $J_{1}=1$ there exist four special values of $J_{2}$, equal to $0,1 / 3,3 / 5$, and 1 . On the other hand, for the fixed value of $J_{2}=1$ the special values of $J_{1}$ are 0 , $1,5 / 3$, and 3 . Consider the predicted behavior of our system [7], if one enlarges the value of temperature, with the fixed value of the magnetic field $h$. For $J_{1}=0$ one has to observe the second order phase transition from the low-temperature Néel antiferromagnetic state to the (paramagnetic) state with $l=0$ for small enough values of $H$, while for large values of $H$ there has to be no phase transitions. For $J_{1}=1$ and $0<J_{2}<5 / 3$ (or for $J_{2}=1$ and $J_{1}>3 / 5$ ), when enlarging temperature one has to observe the phase transition of the first order for small values of the external field $h$, the phase transition of the second order for intermediate values of the external magnetic field, and for large values of the magnetic field there has to be no phase transition. Finally, in the domain of parameters $J_{1}=1$ and $5 / 3<J_{2}<3$ (or for $J_{2}=1$ and $0<J_{1}<3 / 5$ ) for large enough values of the magnetic field there must be no phase transitions. For small values of the external magnetic field $h$ one can observe the second-order phase transition from the Néel phase to the paramagnetic one. If the value of $H$ becomes larger, there exists the possibility that one can observe two second-order phase transitions: with increasing 
temperature the system is transformed from the paramagnetic phase to the antiferromagnetically ordered phase, and, then, again to the paramagnetic phase. For larger values of the magnetic field the theory [7] predicts a first-order phase transition located (along the $T$-axis) before the second-order one (between the Néel phase and the paramagnetic phase). Generally speaking, there exists the possibility of three phase transformations in this domain of parameters: with increasing $T$ one can observe a first-order phase transition followed by two second-order phase transitions (again between the antiferromagnetically ordered and paramagnetic phases). Our numerical solutions for the case $S=1 / 2$ reproduce these results very well. Namely, we compared our numerical results for $T_{N}$ with the ones from the analytical calculations for the critical temperature of the second-order phase transition [7] (we put in their formulas values of $J_{1}, J_{2}, h$ and $T$; notice that they used slightly different notation). We can point out that our numerical results for $T_{N}$ and the results obtained from the analytical approach [7] agree up to the third digit for $T_{N}$. It is important to emphasize that the critical point, at which the antiferromagnetic order parameter becomes zero, is also seen in the temperature behavior of the magnetization: $m$ increases with temperature for $T<T_{N}$ and decreases for $T>T_{N}$. At $T=T_{N}$ the temperature behavior of the magnetization manifests a cusp, hence the magnetic susceptibility of the system has a peculiarity at $T_{N}$. Thus, for small values of the external magnetic field the low-temperature phase is characterized by two nonzero values: $l$ and $m$. Here we have to point out that our numerical scheme does not permit us to study first-order phase transitions in detail: We can only see the presence of such a transition. However, the temperature of the phase transition and the temperatures and fields of stability of phases cannot be obtained within our approach: The behavior of $m$ and $l$ in the region of the first-order phase transition strongly depends on used «initial conditions», and, hence, can be artifacts of approximations of our numerics. It was interesting to study the temperature behavior of $l$ and $m$ for large enough value of the inter-sublattice coupling constant $J_{2}=2$. Totally according to the analytical prediction [7] we found two second-order phase transitions in our numerical calculations: With increasing $T$ the system is transformed from the phase with $l=0$ to the antiferromagnetically ordered phase, and then again to the paramagnetic phase, i.e., the one re-entrant phase transition takes place.

Analytical results are also known for the case, when one changes the value of the external magnetic field, with the fixed value of temperature. For $J_{1}=0$ one has to observe the phase transition from the Néel state to the state with $l=0$ for $T<J_{2} / 4$, and no phase transitions for $T>J_{2} / 4$. If $J_{1}=1$ and $0<J_{2}<5 / 3$ (or for $J_{2}=1$ and $J_{1}>3 / 5$ ) with increasing value of $H$ one has to observe a first-order phase transition from the antiferromagnetic phase to the phase with $l=0$ for small enough temperatures and a second-order phase transition for intermediate temperatures, while for $T>\left(J_{1}+J_{2}\right) / 4$ there must be no phase transformations. Finally, in the domain of values of coupling constants $J_{1}=1$ and $5 / 3<J_{2}<3$ (or for $J_{2}=1$ and $0<J_{1}<3 / 5$ ) for small enough temperatures one has to observe a first-order phase transition from the Néel state to the phase with $l=0$ with increasing $H$. For $T>\left(J_{1}+J_{2}\right) / 4$ there must be no phase transitions. For intermediate range of temperatures it is possible to observe either a second-order or a first-order phase transition, followed by the second-order transition to the phase with $l=0$. These analytical results are also well reproduced by our numerical solutions. The calculated value of the critical magnetic field of the above-discussed second-order phase transition also agrees with the results of analytical calculations (i.e., when we put values of $J_{1}, J_{2}, h$ and $T$ in the formulas of [7]). However the agreement is worse when comparing the values of $T_{N}$; see above: The difference appears already in the third digit for the critical field. For large values of $J_{1}$ we observed metamagnetic first-order phase transitions with jumps of $l$ and $m$. The values of the critical field, calculated within our numerical procedure, agree with the ones, obtained from the analytical formulas of [7], however, again, the agreement is worse, than for $T_{N}$. It turns out that the agreement between the critical values of the external field calculated numerically and analytically is not as good as for the Néel temperature. However, we emphasize that qualitatively the behavior of the antiferromagnetic order parameter as a function of the external magnetic field (and as a function of temperature; see above) agrees very well with the analytical predictions of Refs. 6, 7. Summarizing the case of the lowest crystalline electric field doublet, $S=1 / 2$, we conclude that our numerical procedure of solving mean-field equations for the case of the two-sublattice antiferromagnet reproduces very well known analytical results.

Now we turn to the numerical solutions of mean-field equations for the case with higher values of $S$. Here, unfortunately, there are no analytical results for the temperature and magnetic field dependencies of the antiferromagnetic order parameter $l$ and the total magnetization $m$ of the two-sublattice antiferromagnet in a strong crystalline electric field. Let us consider typical results of our numerical calculations for higher 
values of $S$. We have already checked our numerical procedure for $S=1 / 2$. However, because there are no analytical results for higher values of $S$, we had to develop some additional numerical procedure to check the validity of our solutions for the case of large $S$. We have realized the following checking scheme: Total intervals of possible values for $m$ and $l$ were divided by the finite number of «steps», which formed the finite number of values of $m$ and $l$ taking part in our checking procedure. These values were combined into a definite number of pairs of possible values $m$ and $l$. Those pairs were set into our Eqs. (3). Then, the differences between the «left» and «right» parts of given equations for all pairs of values $m$ and $l$ were compared with each other, and the pair with the smallest difference was named the «solution» the set of equations for given values of all the parameters considered. Evidently, this checking procedure is approximate (its accuracy depends on the size of the abovementioned «steps»), but it is also the most reliable and evident method of searching solutions of the considered equations. As a result of these calculations, one can obtain the dependencies of $l$ and $m$ of $T$ or $h$, which must correspond to the results of our previous calculations. Besides, if there exist, for example, two possible solutions, for definite values of all parameters, this procedure gives us both solutions, as it was obtained by us, and we must choose a branch of functions for $l$ and $m$ with the expected behavior of the accepted model in given case. Indeed, the results, we have obtained by such a checking, in the limits of the accepted accuracy, confirm the results obtained by the «standard» calculation procedure, which we have used before for all our calculations, including the case $S=1 / 2$.

Basically, the behavior of $S \geq 1$ system is similar to the abovepresented case of the crystalline electric field doublet $(S=1 / 2)$. However, there exist some very important differences, which, in such a way, manifest a peculiar feature of the case of higher values of $S(S>1 / 2)$. For instance, Fig. 1 shows the typical temperature behavior of the values of the order parameter $l$ and the magnetization $m$ for $S=3 / 2$ for a high value of the magnetic field $H=4.5$ for $J_{1}=1$ and various values of the inter-sublattice constants $J_{2}=0.5,1,1.5,2,2.5,3$. It is clear from Fig. 1 that for small values of $J_{2}=0.5,1,1.5$ the system is in the paramagnetic phase, with the smooth decrease of $m$ with the growth of $T$. For $J_{2}=2.5$ we can observe the phase transition between the antiferromagnetic and paramagnetic phases (at low temperatures). For large value of $J_{2}=3$ we see the second-order phase transition between phases with $l \neq 0$ and $l=0$. The most interesting behavior is seen for $J_{2}=3$. Here, together with the second-order phase transition between para-
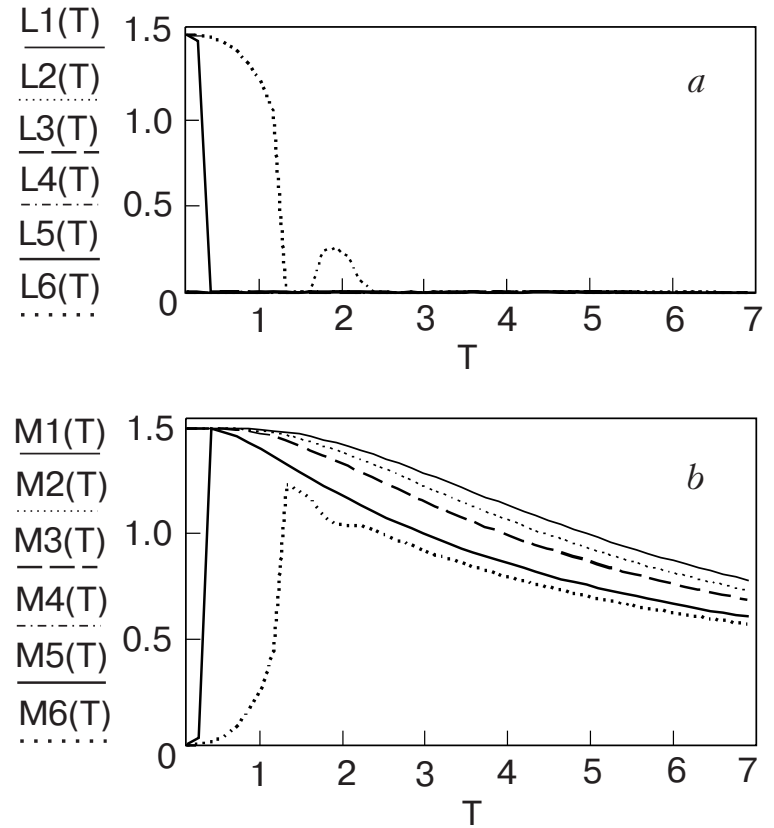

Fig. 1. Temperature dependence of the vector of antiferromagnetism $l(a)$ and the magnetization $m(b)$ for the model with $S=1.5$ for $J_{1}=1$ and $H=4.5$. The lines are shown for $J_{2}=0.5$ (L1, M1); $J_{2}=1$ (L2, M2); $J_{2}=1.5$ (L3, M3); $J_{2}=2$ (L4, M4); $J_{2}=2.5$ (L5, M5); $J_{2}=3$ (L6, M6). Notice that the line for $m(T)$ for $J_{2}=2$ practically coincides with the one for $J_{2}=1.5$. For $J_{2}=0.5,1,1.5,2$ we have basically zero order parameter, $l=0$, for the total considered temperature interval.

magnetic and Néel phases at high temperatures ( $T \sim 2.4$ ), one can see additional features in the temperature dependencies of $l$ and $m$. With these values of the external magnetic field at low temperatures the system is in the antiferromagnetic phase, then it experiences the phase transition to the state with $l=0$ (at $T \sim 1.3$ ), and then, with the further increase of the temperature the system returns to the phase with $l \neq 0$ (at $T \sim 1.7$ ). The onset of such additional phase transitions distinguishs the system with $S>1 / 2$ from those with lowest doublets $(S=1 / 2)$. We point out again, that our method of calculations does not give the opportunity to find characteristics of the first-order phase transitions (which can also take place for different values of the coupling constants and the external magnetic field). Because of this reason we do not present our numerical solutions for $m(T)$ and $l(T)$ in those regions of parameters.

We also calculated the dependence of the vector of antiferromagnetism and the magnetization on the value of the external magnetic field $h$. Jumps and cusps are present in the magnetic field behavior, which are characteristic for the metamagnetic phase transitions, similar to what is known for the ground state for $S=1 / 2$ [8]. As for the doublet case, at 
smaller values of coupling constants the first-order phase transitions between the antiferromagnetic (low field) phase and the paramagnetic phases is replaced by the second-order phase transitions. Other magnetic field and temperature behavior for $S \geq 1$ qualitatively coincide with the discussed above behavior for the case $S=1 / 2$. For example, the results for the magnetic field behavior for $J_{1}=1$ and $J_{2}=0.5,1,1.5,2,2.5,3$ intra- and inter-sublattice coupling constants for high enough temperature $T=1.5$ are presented in Fig. 2 . For $J_{2}=0.5,1$ the system is in the paramagnetic phase. For $J_{2} \leq 1.5$, second-order phase transitions between the low-field antiferromagnetic phase and high-field paramagnetic phase take place. One can see how the features of the second-order phase transitions between the Néel ordered phase and paramagnetic phase are developed with the growth of the inter-sublattice couipling constant $J_{2}$. For higher values of $J_{2}$ (or lower values of the temperature) second-order phase transition between the antiferromagnetic and paramagnetic phases is replaced by the first-order phase transition (we also do not show these results, because of the reasons, discussed above). Similar behavior is manifested by the numerical mean-field solutions for other values of $J_{1}$ and $J_{2}$ and $S$.
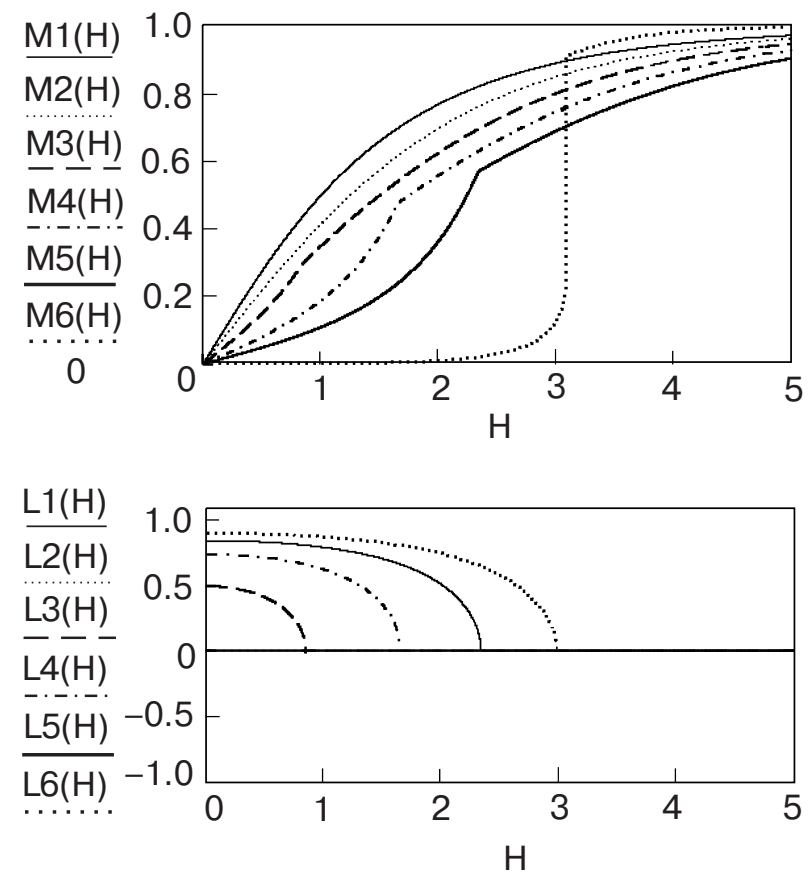

Fig. 2. Magnetic field dependence of the vector of antiferromagnetism $l(a)$ and the magnetization $m(b)$ for the model with $S=1$ for $J_{1}=1$ and $T=1.5$. The lines are shown for $J_{2}=0.5$ (L1, M1); $J_{2}=1$ (L2, M2); $J_{2}=1.5$ (L3, M3); $J_{2}=2$ (L4, M4); $J_{2}=2.5$ (L5, M5); $J_{2}=3$ (L6, M6). For $J_{2}=0.5,1$ we have basically zero order parameter, $l=0$, for all considered values of the external magnetic field.
Let us now discuss obtained results, to emphasize on some regular features in the behavior of the order parameter and the total magnetization of the considered systems. The agreement between our numerical results and previously obtained analytical ones (for $S=1 / 2$ ) is very good for the values of the Néel temperature, and less good for the critical values of the external magnetic field (for higher values of $S$ there were no analytical results previously). In general, the temperature and magnetic field behavior of the two-sublattice antiferromagnets with the large values of low-lying multiplets caused by the strong crystalline electric field is similar to the behavior in the case $S=1 / 2$. For small values of the external magnetic field, or for large values of the temperature the second-order phase transitions between the ordered antiferromagnetic phase and the paramagnetic phase with $l=0$ take place. For intermediate values of the magnetic field and the temperature the firstorder metamagnetic phase transitions can be observed (cf. [6,7]). It turns out, however, that for $S \geq 1$ more than one phase transitions can be seen in the temperature dependencies of both the antiferromagnetic order parameter and the total magnetization. In some cases (for large enough inter-sublattice couplings and high values of the external magnetic field) they are re-entrant phase transitions between the antiferromagnetically ordered phase(s) and paramagnetic phase(s), as for the case $S=1 / 2$. However, in contrast to the situation of lowest crystalline electric field doublets $(S=1 / 2)$, the low-temperature phase is antiferromagnetically ordered, and these re-entrant phase transformations are doubled. Such double re-entrant phase transitions are shown in Fig. 3, where the (approximate) illustration of the $H-T$ phase diagram is presented for $S \leq 1$ for such values of inter- and intra-sublattice coupling constants $\left(J_{1}\right.$ and $\left.J_{2}\right)$, for which only second-order phase transitions take place.

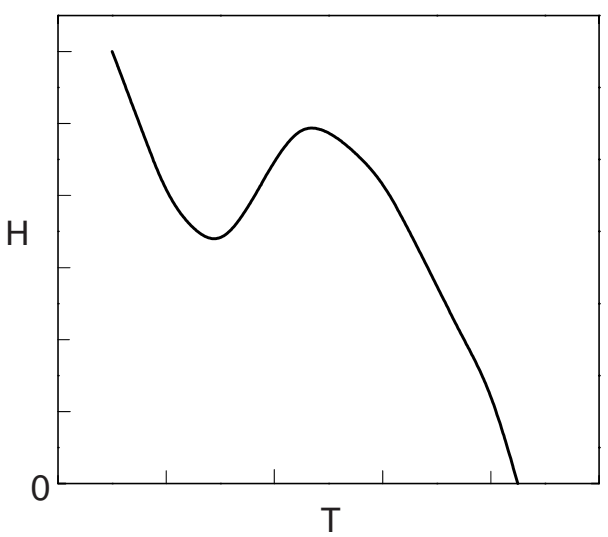

Fig. 3. The illustration of the $H-T$ phase diagram for the two-sublattice antiferromagnet in the strong crystalline electric field for $S \geq 1$ for large values of $J_{2}$ with the series of re-entrant phase transitions. 
[We emphasize again that our numerical procedure only permits us to speak about the existence of the first-order phase transitions, but does not permit to obtain correct values of the critical temperatures and fields. This is why we do not present phase diagrams for the values of $J_{1}$ and $J_{2}$ for which first-order phase transitions can take place.] Observe that for small values of $T$ the mean-field solution can produce invalid results, and, therefore, we do not present the low-temperature part of the phase diagram. One can see, that if the magnetic field is large enough, when one increases the temperature, the system is transformed from the antiferromagnetically ordered phase to the paramagnetic phase, then, with further growth of temperature the system is transformed to the antiferromagnetic Néel phase, and, finally, it goes to the (magnetically disordered) phase with $l=0$ at high temperatures. Notice that the phase transition from the low temperature antiferromagnetically ordered phase to the paramagnetic phase can be of the first order also (for different values of coupling parameters $J_{1}$ and $J_{2}$ ). Such a possibility of the series of re-entrant phase transitions between the Néel antiferromagnetically ordered and paramagnetic phases qualitatively differs our study from previous results [6-8].

Recently several new phase transitions between antiferromagnetically ordered and paramagnetic phases with different values of the vector of the antiferromagnetism were observed in rare-earth compound $\mathrm{YbRh}_{2} \mathrm{Si}_{2}[12,13]$. That compound is characterized by one of the lowest Néel temperature for rare-earth systems $\left(T_{N}=70 \mathrm{mK}\right)$, which permits to study phase transformations, induced by the external magnetic field. On the other hand, the compound is characterized by a strong crystalline electric field. In that compound additional peculiarities were also observed in the magnetic field behavior of its characteristics [14]. It is possible that the features of those new phase transitions in that rare-earth compound are related to the phases, studied theoretically in our work. We point out that in this compound the magnetic anisotropy is not of the «easy-axis» but rather of the «easy-plane» type. This is why we cannot compare our results with the data of experiments directly. We believe, however, that the re-entrant phase transitions, found theoretically in our study, are characteristic for many metamagnets, and can be observed in many rare-earth based antiferromagnets, as well as in transition-metal-based antiferromagnets with the large «easy-axis» magnetic anisotropy and high values of their spins in high enough magnetic fields.

In conclusion, in this study we have solved numerically the set of self-consistent equations which describe the mean-field solution a two-sublattice anti- ferromagnet with the strong «easy-axis» crystalline electric field. Such a consideration has to be valid for rare-earth antiferromagnets, in which the energy difference between the lowest multiplets and the higher ones due to the crystalline electric field are larger than the values of the inter-moment couplings and the value of the external magnetic field for small enough temperatures. Our calculations reveal several new phase transitions between the antiferromagnetic (Néel) and paramagnetic phases. In the limiting case of the lowest crystalline electric field doublet, $S=1 / 2$, our results coincide with the previously known analytical ones. We point out nonmonotonous dependencies of the total magnetization of the system as the function of temperature, with the critical behavior of the magnetic susceptibility at phase transitions to the antiferromagnetic phases. New additional double re-entrant phase transitions appear to be more pronounced for higher degeneracy of the low-lying multiplets caused by the crystalline electric field $(S \leq 1)$ of magnetic ions. For higher values of the magnetic field phase transitions of the first-order (with jumps of the magnetization and/or the vector of antiferromagnetism) are transformed to second-order phase transitions, and, with the further increase of the field value the phase transitions disappear (the dependencies of $m$ and $l$ become smooth). Additional phase transitions take place for higher values of the degeneracy of the lowest crystalline electric field multiplet. Our predictions have to be valid for Ising-like two-sublattice magnetic systems consisting of spins with the values larger than $1 / 2$. We believe that some of re-entrant phase transitions, studied in our theory, were observed in rare-earth compounds with low values of the Néel temperature, and, probably, similar phase transitions can be observed in the transition metal compounds also.

1. L. Néel, Ann. Phys. 17, 61 (1932).

2. L.D. Landau, Phys. Zs. UdSSR 4, 675 (1933).

3. C.G. Shull, W.A. Strauser, and E.O. Wollan, Phys. Rev. 83, 333 (1951).

4. P. Weiss, J. Phys. Radium 6, 661 (1907).

5. S.V. Vonsovskii, Magnetism, Nauka, Moscow (1971) (in Russian).

6. K. Motizuki, J. Phys. Soc. Jpn. 14, 759 (1959).

7. V.G. Bar'yakhtar, I.M. Vitebskii, and D.A. Yablonskii, Fiz. Tv. Tela 19, 2135 (1977) (in Russian).

8. A.K. Zvezdin, V.M. Matveev, A.A. Mukhin, and A.I. Popov, Rare-Earth Ions in Magnetically Ordered Crystals, Nauka, Moscow (1985) (in Russian).

9. J.H. Van Vleck, Proc. Nat. Acad. Am. 12, 662 (1926).

10 J.H. Van Vleck, Phys. Rev. 29, 727 (1927); ibid. 30, 31 (1927); ibid. 31, 587 (1928). 
11. V.V. Eremenko and V.A. Sirenko, Magnetic and Magneto-Elastic Properties of Antiferromagnets and Superconductors, Kiev, Naukova Dumka (2004) (in Russian).

12. J. Plessel, M.M. Abd-Elmeguid, J.P. Sanchez, G. Knebel, O. Trovarelli, and F. Steglich, Phys. Rev. B67, 180403 (2003).
13. G. Dionicio, H. Wilhelm, G. Sparn, J. Ferstl, C. Geibel, and F. Steglich, Physica B359, 50 (2005).

14. J. Custers, P. Gegenwart, H. Wilhelm, K. Neumaier, Y. Tokiwa, O. Trovarelli, C. Geibel, F. Steglich, C. Pepin, and P. Coleman, Nature 424, 524 (2003). 